\title{
Traveling solar-wind bulk-velocity fluctuations and their effects on electron heating in the heliosphere
}

\author{
Hans J. Fahr ${ }^{1}$, Igor V. Chashei ${ }^{2}$, and Daniel Verscharen ${ }^{3}$
}

\author{
1 Argelander Institute for Astronomy, University of Bonn, Auf dem Hügel 71, 53121 Bonn, Germany \\ e-mail: hfahr@astro.uni-bonn.de \\ ${ }^{2}$ Lebedev Physical Institute, Leninskii Prospect 53, 117924 Moscow, Russia \\ 3 Space Science Center, University of New Hampshire, 8 College Road, Durham NH 03824, USA
}

Received 17 June 2014 / Accepted 12 September 2014

\begin{abstract}
Ambient plasma electrons undergo strong heating in regions associated with compressive bulk-velocity jumps $\Delta U$ that travel through the interplanetary solar wind. The heating is generated by their specific interactions with the jump-inherent electric fields. After this energy gain is thermalized by the shock passage through the operation of the Buneman instability, strong electron heating occurs that substantially influences the radial electron temperature profile. We previously studied the resulting electron temperature assuming that the amplitude of the traveling velocity jump remains constant with increasing solar distance. Now we aim at a more consistent view, describing the change in jump amplitude with distance that is caused by the heated electrons. We describe the reduction of the jump amplitude as a result of the energy expended by the traveling jump structure. We consider three effects: energy loss due to heating of electrons, energy loss due to work done against the pressure gradient of the pick-up ions, and an energy gain due to nonlinear jump steepening. Taking these effects into account, we show that the decrease in jump amplitude with solar distance is more pronounced when the initial jump amplitude is higher in the inner solar system. Independent of the initial jump amplitude, it eventually decreases with increasing distance to a value of about $\Delta U / U \simeq 0.1$ at the position of the heliospheric termination shock, where $\Delta U$ is the jump amplitude, and $U$ is the average solar-wind bulk velocity.The electron temperature, on the other hand, is strongly correlated with the initial jump amplitude and leads to electron temperatures between $6000 \mathrm{~K}$ and $20000 \mathrm{~K}$ at distances beyond 50 AU. We compare our results with in situ measurements of the electron-core temperature from the Ulysses spacecraft in the plane of the ecliptic for $1.5 \mathrm{AU} \leq r \leq 5 \mathrm{AU}$, where $r$ is the distance from the Sun. Our results agree very well with these observations, which corroborates our extrapolated predictions beyond $r=5 \mathrm{AU}$.
\end{abstract}

Key words. plasmas - Sun: heliosphere - solar wind

\section{Introduction}

The electron temperature in the solar wind is expected to rapidly decrease with increasing distance $r$ from the Sun, as soon as the electron heat conduction ceases that serves as the prime energy source (Feldman et al. 1975; Pilipp et al. 1987; Scime et al. 1994). At distances smaller than 5 AU, electron distribution functions have been identified as core-halo-structured distributions with an electron heat flux that decreases with a power law according to $\propto r^{-2.36}$ (see McComas et al. 1992). On the basis of electron data taken from the Helios, Wind, and Ulysses spacecraft, Maksimovic et al. (2005) have carefully analyzed the radial change of the core-halo-strahl structure of the electron distribution function with distance from the Sun in the range between $0.3 \mathrm{AU}$ and 1.5 $\mathrm{AU}$. These authors found that while the relative abundance of core electrons remains fairly constant with distance, the relative abundance of halo electrons increases and that of strahl electrons decreases, suggesting that the relative increase in halo electrons is connected to the relative loss in strahl electrons. Interestingly, however, both the core electron temperature and the halo electron temperature decrease with distance. This effect can be represented by kappa distribution functions with decreasing kappa-indices and will be best fitted by the decrease of the electron kappa index from $\kappa=6$ at $r=0.5 \mathrm{AU}$ to $k=3$ at $r=1.5 \mathrm{AU}$. The increase in the relative abundance of the halo population is interpreted as the consequence of an isotropization of the strahl population, which leads to a conversion into the halo population (see also Štverák et al. 2009).

Beyond the outer ranges of the Ulysses trajectory (i.e., at solar distances beyond $5 \mathrm{AU}$ ), measurements of low-energy solarwind electrons are not available. Up to now, electron temperatures have been expected to decrease to negligible values in this region for theoretical reasons. At such large distances from the Sun, processes such as whistler-wave-turbulence generation caused by instabilities that are driven by the electron heat flux (see Scime et al. 1994; Gary et al. 1994) become unimportant. Pitch-angle scattering and energy-diffusion processes can likewise be neglected at these distances (Schlickeiser et al. 1991; Achatz et al. 1993). However, more recently, Breech et al. (2009) have presented a theoretical study of the heating of solarwind protons and electrons by dissipation of magnetohydrodynamic (MHD) turbulent energy. While their study shows that the theoretically obtained proton temperatures fit the Ulysses data, the theoretically obtained electron temperatures (see Fig. 3 in Breech et al. 2009), fail to fit almost all the data because they are too low.

As a remedy of that failure, we most recently conjectured that the interaction between electrons and the electric fields associated with traveling fluctuations in the solar-wind bulk velocity (i.e., traveling shocks) can provide an energy source for electron heating in this part of the heliosphere (Chashei \& Fahr 2014). All solar-wind properties, including the solar-wind bulk 


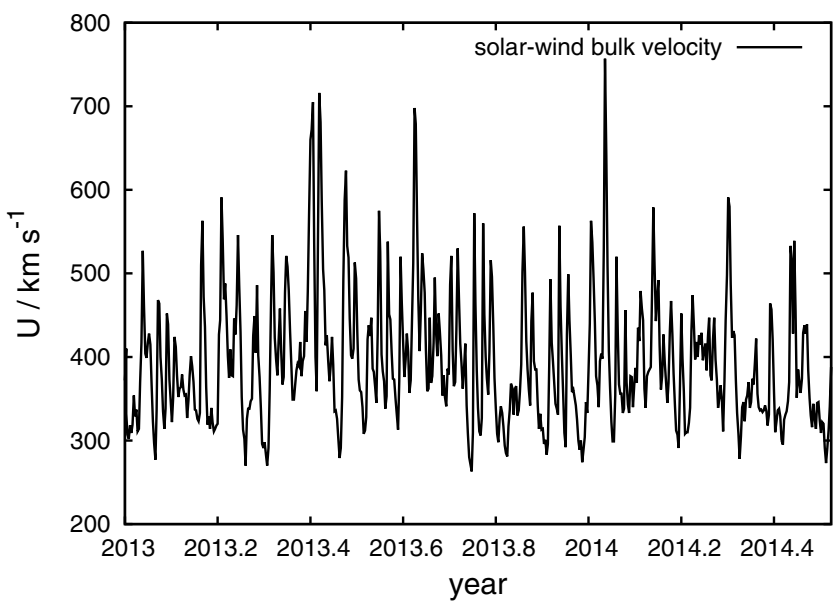

Fig. 1. Solar-wind bulk velocity as a function of time in the plane of the ecliptic at $1 \mathrm{AU}$. We show OMNI-2 data (from $\mathrm{ftp}: / / \mathrm{spdf}$.gsfc. nasa.gov/pub/data/omni/low_res_omni/) from a combined set of spacecraft observations to demonstrate the ubiquitous fluctuations in the solar-wind bulk velocity.

velocity $U$, show strongly pronounced variations on many time scales as well as shock-like structures (Feng et al. 2009; Yue \& Zong 2011; Janvier et al. 2014). We show a time line of the measured solar-wind bulk velocity in the plane of the ecliptic at 1 AU in Fig. 1 (cf. Echer et al. 2005; Lai et al. 2010; Sokół et al. 2013). In agreement with these observations, we find a typical occurrence rate of about 30 jumps with a significant amplitude per year. These jumps are convected over the spacecraft with an average solar-wind bulk velocity of $U \approx 400 \mathrm{~km} \mathrm{~s}^{-1}$, which leads to a typical distance of about $L_{\mathrm{j}}=3 \mathrm{AU}$ between subsequent shocks.

Fluctuations $\Delta U(t) \equiv U(t)-\langle U(t)\rangle$, where $\langle\cdot\rangle$ is the time average and $U$ is the solar-wind bulk velocity, persist to large distances from the Sun up to 20 to $40 \mathrm{AU}$, as clearly demonstrated by Voyager observations (Richardson et al. 1995). Figure 2 in Richardson et al. (1995) shows that while the bulk velocity fluctuations survive up to large solar distances, the average bulk velocity $U=\langle U(t)\rangle$ appears to be constant, implying that differential kinetic energy is not converted into kinetic energy of the wind, but into thermal degrees of freedom of the plasma system.

We suspect that these bulk-velocity fluctuations are responsible for the still poorly understood heating of electrons at larger distances from the Sun. We have recently proposed in a quantitative discussion that compressional bulk-velocity waves heat solar-wind electrons (Chashei \& Fahr 2014). We determined the fraction of the differential kinetic energy of the traveling shocks and quantified the energy that is transferred to thermal energy of the solar-wind electrons by means of the Buneman instability as a function of the bulk speed $U_{2}$ downstream of the velocity jump. The joint bulk speed of electrons and protons, after passing the jump-associated electric-field jump, is given by

$m_{\mathrm{e}} U_{2 \mathrm{e}}+m_{\mathrm{p}} U_{2 \mathrm{p}}=\left(m_{\mathrm{e}}+m_{\mathrm{p}}\right) U_{2}$,

where $m_{\mathrm{e}, \mathrm{p}}$ denotes the mass of the electron and of the proton, and $U_{2}$ denotes the bulk velocity of the downstream centerof-mass system. With $m_{\mathrm{e}} \ll m_{\mathrm{p}}$, this expression leads to (see Chashei \& Fahr 2014)

$U_{2} \approx U_{2 \mathrm{p}}+\frac{m_{\mathrm{e}}}{m_{\mathrm{p}}} U_{2 \mathrm{e}} \approx U_{2 \mathrm{p}}+s \sqrt{\frac{m_{\mathrm{e}}}{m_{\mathrm{p}}}} U_{2 \mathrm{p}}$ where $s \equiv(U+\Delta U) /(U-\Delta U)$ is the jump compression ratio. The difference $U_{2}-U_{2 \mathrm{p}}$ is much smaller than $U_{2 \mathrm{p}}$, and hence the overshoot energy of the electrons in the downstream bulk frame is given by

$\Delta W_{\mathrm{e}}=\frac{1}{2} \frac{m_{\mathrm{e}} m_{\mathrm{p}}}{m_{\mathrm{e}}+m_{\mathrm{p}}}\left(U_{2 \mathrm{e}}-U_{2 \mathrm{p}}\right)^{2} \approx \frac{1}{2} m_{\mathrm{e}} U_{2 \mathrm{e}}^{2}$.

If this kinetic energy $\Delta W_{\mathrm{e}}$ of the overshooting electrons can be locally converted into electron heat, this process leads to an electron temperature increase $\Delta T_{\mathrm{e}}$ after each jump passage given by

$\Delta T_{\mathrm{e}}=\frac{m_{\mathrm{e}} U_{2 \mathrm{e}}^{2}}{3 k}=\frac{m_{\mathrm{p}} \Delta U^{2}}{3 k}\left(1-\frac{1}{s^{2}}\right)$,

where $k$ is the Boltzmann constant.

This process describes an average gain of thermal energy that leads to a systematic heating of the solar-wind electrons per radial increment $\mathrm{d} r$ as a result of repeated shock passages. The resulting radial dependence of the electron temperature can be described by a transport equation for the thermal energy. We expect that the electron heating due to accumulated jump passages in the heliosphere beyond about $5 \mathrm{AU}$ is statistical in nature. We denote the average distance between consecutive jumps as $L_{\mathrm{j}}$ and define the average jump occurrence rate as $v_{\mathrm{j}} \equiv U / L_{\mathrm{j}}$. With these definitions, the equation for the radial electron temperature is given in the following differential form (see Chashei \& Fahr 2014):

$\frac{\mathrm{d} T_{\mathrm{e}}}{\mathrm{d} r}+2 \frac{T_{\mathrm{e}}}{r}=\Delta T_{\mathrm{e}} \frac{\Delta U}{L_{\mathrm{j}} U}=\Delta T_{\mathrm{e}} \frac{\Delta X}{L_{\mathrm{j}}}$,

where $\Delta X \equiv \Delta U / U$. The term on the right-hand side of Eq. (5) describes the electron heating induced by jump passages. When they solved this equation, Chashei \& Fahr (2014) assumed that $\Delta T_{\mathrm{e}}=\Delta T_{\mathrm{e}}(\Delta U)$ is a constant. This assumption is true if $\Delta U$ is independent of the distance $r$. In that case, the radial profile of the resulting electron temperature is given by

$T_{\mathrm{e}}(x)=\frac{1}{x^{2}}\left(x^{3} \frac{m_{\mathrm{p}} U^{2} \Delta X^{3}}{18 k} \frac{r_{0}}{L_{\mathrm{j}}}+T_{\mathrm{e} 0}\right)$,

where $x \equiv r / r_{0}$ is the dimensionless spatial coordinate and $T_{\mathrm{e} 0}$ denotes the electron temperature at $r=r_{0}=1 \mathrm{AU}$ (solution shown in Fig. 1 of Chashei \& Fahr 2014). This solution suffers from the inconsistency that the jump kinetic energy is assumed to be constant even after transferring energy to the electrons. We make this earlier approach more consistent in the sections below by taking into account the energy consumption at the passage of each jump during this process.

\section{Change of the jump amplitude with distance from the Sun}

To increase the consistency of our approach, we now include higher-order corrections to the electron heating due to the variation of the jump amplitude $\Delta U$ with distance $r$. This amplitude is assumed to be the primary physical reason for the gain in thermal energy of the electrons. Therefore, we have to adequately describe the change of $\Delta U$ caused by energy expended by the excess kinetic energy of the jump structure. In situ observations by the Voyager 2 spacecraft at distances between $10 \mathrm{AU}$ and $40 \mathrm{AU}$ from the Sun (Richardson et al. 1995) showed that compared 
H. J. Fahr et al.: Bulk-velocity fluctuations and electron heating in the heliosphere

with solar-wind bulk-velocity measurements carried out simultaneously at 1 AU by IMP 8 , the average solar-wind speed does not change with distance. On the other hand, the amplitude of the speed fluctuations strongly decreases with distance from the Sun (see Fig. 6 of Richardson et al. 1995). This observation indicates that these fluctuations do work, while the bulk-solar-wind outflow does not. Our theoretical approach is based on these observations, adopting that the average solar-wind speed $U$ is constant with distance from the Sun. Based on this observationally supported assumption, we consider three effects that determine the change of $\Delta U$ with distance $r$ :

a) heating of electrons,

b) work done against the slower side of the jump with its higher pick-up-ion pressure, and

c) steepening of the jump profile by nonlinear superpositions of small-scale bulk-velocity fluctuations.

In the following, we separately discuss these three different effects.

\subsection{Reduced compression due to electron heating}

We consider the spatial divergence of the jump-associated flow of excess kinetic energy on the high-velocity side of a jump with the amplitude $\Delta X=\Delta U / U$. This jump acts as a local source of electron thermal energy, and this heating reflects a local energy sink for the excess kinetic energy that is represented by the compression profile $\Delta X(r)$. Using Eq. (5) for the electron temperature, we can formulate an expression for the energy sink associated with this jump as the divergence of the excess kinetic energy flow:

$\frac{1}{r^{2}} \frac{\mathrm{d}}{\mathrm{d} r} r^{2}\left(U \frac{1}{2} n_{\mathrm{e}} m_{\mathrm{p}} \Delta U^{2}\right)=-\frac{3}{2} U k \Delta T_{\mathrm{e}} n_{\mathrm{e}} \frac{\Delta U}{L_{\mathrm{j}} U}$,

where $n_{\mathrm{e}}=n_{\mathrm{p}}=n$ is the local solar-wind electron/proton number density and $\Delta T_{\mathrm{e}}$ is the electron temperature increase per jump passage as given by Eq. (4). We assume that the mean bulk velocity $U=(1 / 2)(U+\Delta U+U-\Delta U)$ is constant and find

$\frac{\mathrm{d}}{\mathrm{d} r}\left(\Delta U^{2} n_{\mathrm{e}}\right)+\frac{2}{r}\left(\Delta U^{2} n_{\mathrm{e}}\right)=-3 \frac{k \Delta T_{\mathrm{e}} n_{\mathrm{e}}}{m_{\mathrm{p}} U} \frac{\Delta U}{L_{\mathrm{j}}}$.

Supported by Voyager data, we assume that the traveling jumps in bulk velocity have a small amplitude $(\Delta U \ll U$, which is equivalent to $\Delta X \ll 1$ ). This observation allows us to approximate the electron-temperature increase per jump passage in Eq. (4) using the linearizations

$s=\frac{1+\Delta X}{1-\Delta X} \simeq 1+2 \Delta X$

and

$s^{2} \simeq(1+2 \Delta X)^{2} \simeq 1+4 \Delta X$.

We can then rewrite Eq. (4) as

$\Delta T_{\mathrm{e}} \simeq \frac{m_{\mathrm{p}} \Delta U^{2}}{3 k}[1-(1-4 \Delta X)]=\frac{4 m_{\mathrm{p}} U^{2} \Delta X^{3}}{3 k}$

and obtain from Eq. (8)

$\frac{2}{\Delta X} \frac{\mathrm{d} \Delta X}{\mathrm{~d} r}+\frac{1}{n_{\mathrm{e}}} \frac{\mathrm{d} n_{\mathrm{e}}}{\mathrm{d} r}+\frac{2}{r}=-4 \frac{\Delta X^{2}}{L_{\mathrm{j}}}$.

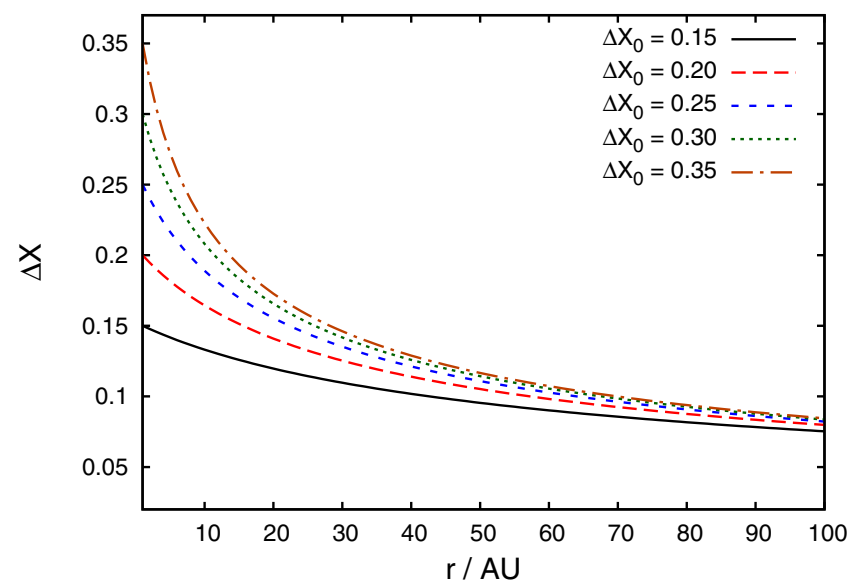

Fig. 2. Compression $\Delta X$ as a function of distance $r$ from the Sun for five different values of $\Delta X_{0}$ at $r_{0}=1 \mathrm{AU}$ with $r_{0} / L_{\mathrm{j}}=1 / 3$. The compression decreases with distance and approaches a value of about 0.1 at the position of the termination shock. The dependence on the jump occurrence $r_{0} / L_{\mathrm{j}}$ is discussed in Chashei \& Fahr (2014).

Assuming a spherically symmetric decrease in density of the average solar wind flow with $n_{\mathrm{e}} \propto r^{-2}$, we then obtain

$\frac{2}{\Delta X} \frac{\mathrm{d} \Delta X}{\mathrm{~d} r}=-4 \frac{\Delta X^{2}}{L_{\mathrm{j}}}$

and find

$\frac{\mathrm{d} \Delta X^{-2}}{\mathrm{~d} r}=4 \frac{1}{L_{\mathrm{j}}}$

From this relation, we derive in a first step

$\left|\Delta X^{-2}\right|_{r_{0}}^{r}=4 \frac{1}{L_{\mathrm{j}}} \int_{r_{0}}^{r} \mathrm{~d} r=\frac{4}{L_{\mathrm{j}}}\left(r-r_{0}\right)$,

which delivers a solution of the form

$\Delta X=\frac{1}{\sqrt{\Delta X_{0}^{-2}+\frac{4}{L_{\mathrm{j}}}\left(r-r_{0}\right)}}=\frac{\Delta X_{0}}{\sqrt{1+\frac{4 r_{0}}{L_{\mathrm{j}}} \Delta X_{0}^{2}(x-1)}}$.

We show $\Delta X$ as a function of distance $r$ from the Sun for five different values of $\Delta X_{0}$ in Fig. 2. We use $r_{0} / L_{\mathrm{j}}=1 / 3$ in agreement with observations at $1 \mathrm{AU}$ (see Fig. 1). The jump amplitude $\Delta X$ remarkably decreases with increasing distance from the Sun. The decrease is even more pronounced for cases in which the initial value $\Delta X_{0}$ is higher in the inner solar system. However, independent of $\Delta X_{0}$, the jump amplitude assumes values of $\lesssim 0.1$ at the position of the heliospheric termination shock (i.e., at $r \approx 90 \mathrm{AU})$.

With this result for the dependence of $\Delta X$ on $r$, we solve the earlier differential equation, Eq. (5), for the resulting electron temperature and obtain

$\frac{\mathrm{d} T_{\mathrm{e}}}{\mathrm{d} x}+2 \frac{T_{\mathrm{e}}}{x}=\frac{4}{3} \frac{m_{\mathrm{p}} U^{2}}{k \lambda} \frac{\Delta X_{0}^{4}}{\left(1+4 \Delta X_{0}^{2} \frac{x-1}{\lambda}\right)^{2}}$ 


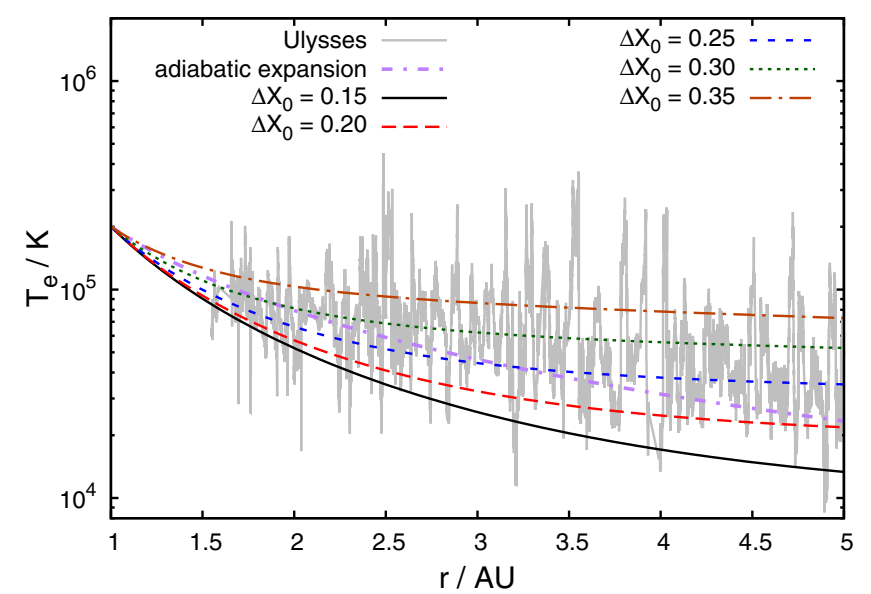

Fig. 3. Electron temperature $T_{\mathrm{e}}$ as a function of distance $r$ from the Sun for five different values of $\Delta X_{0}$ with $r_{0} / L_{\mathrm{j}}=1 / 3$ and $U=400 \mathrm{~km} \mathrm{~s}^{-1}$. The electron temperature is greater than adiabatic. In addition, we show Ulysses observations of the in-situ electron-core temperature in the plane of the ecliptic and the prediction from adiabatic expansion.

where $\lambda \equiv L_{\mathrm{j}} / r_{0}$. The solution of this inhomogeneous differential equation is given by

$$
\begin{aligned}
T_{\mathrm{e}}(x)= & \frac{1}{x^{2}}\left\{\frac{4}{3} \frac{m_{\mathrm{p}} U^{2}}{k \lambda} \Delta X_{0}^{4} \int_{1}^{x} \frac{y^{2}}{[1+a(x-1)]^{2}} \mathrm{~d} y+T_{\mathrm{e} 0}\right\} \\
= & \frac{1}{x^{2}}\left\{\frac{4}{3} \frac{m_{\mathrm{p}} U^{2}}{k \lambda a^{3}} \Delta X_{0}^{4}[2(a-1) \ln [1+a(x-1)]\right. \\
& \left.\left.+\frac{a(x-1)\left[1+a(x-1)+(a-1)^{2}\right]}{1+a(x-1)}\right]+T_{\mathrm{e} 0}\right\}
\end{aligned}
$$

with $a=4 \Delta X_{0}^{2} / \lambda$. We show the result of Eq. (18) for five different values of $\Delta X_{0}$ in Figs. 3 and 4 . We use $T_{\mathrm{e} 0}=2 \times 10^{5} \mathrm{~K}$ and $U=400 \mathrm{~km} \mathrm{~s}^{-1}$. In Fig. 3, we show our results for $1.5 \mathrm{AU} \leq$ $r \leq 5 \mathrm{AU}$ and compare them with in situ Ulysses measurements of the electron-core temperature during the spacecraft's first orbit in the plane of the ecliptic (from December 28, 1990 until December 31, 1991). The data were taken with the Ulysses SWOOPS experiment (Bame et al. 1992). Our predictions and in situ measurements agree very well. The modeled and observed electron temperatures are significantly higher than predicted for an adiabatically expanding gas (i.e., $T_{\mathrm{e}} \propto r^{-4 / 3}$ ). We achieve the best agreement for values of $\Delta X_{0}$ between 0.25 and 0.3 . In Fig. 4 , we extrapolate our results beyond $5 \mathrm{AU}$ and show our predictions for $1 \mathrm{AU} \leq r \leq 100 \mathrm{AU}$. The electron temperature in our model is significantly higher than predicted from adiabatic expansion beyond $10 \mathrm{AU}$ for all values of $\Delta X_{0}$. It assumes values of about $T_{\mathrm{e}} \approx 10^{4} \mathrm{~K}$ at the position of the heliospheric termination shock.

\subsection{Change of compression due to work done against entropized pick-up ions}

In this section, we consider another effect that may also contribute to a decrease in the jump amplitude $\Delta X$ : the work done by the faster front against the difference in ion pressure over the traveling shock front. The faster regime $\left(U_{1}=U+\Delta U\right)$ is running into the slower regime $\left(U_{2}=U-\Delta U\right)$ with a differential velocity $\Delta U$. During this process, the plasma has to do work against the pressure difference between the two regimes to adapt

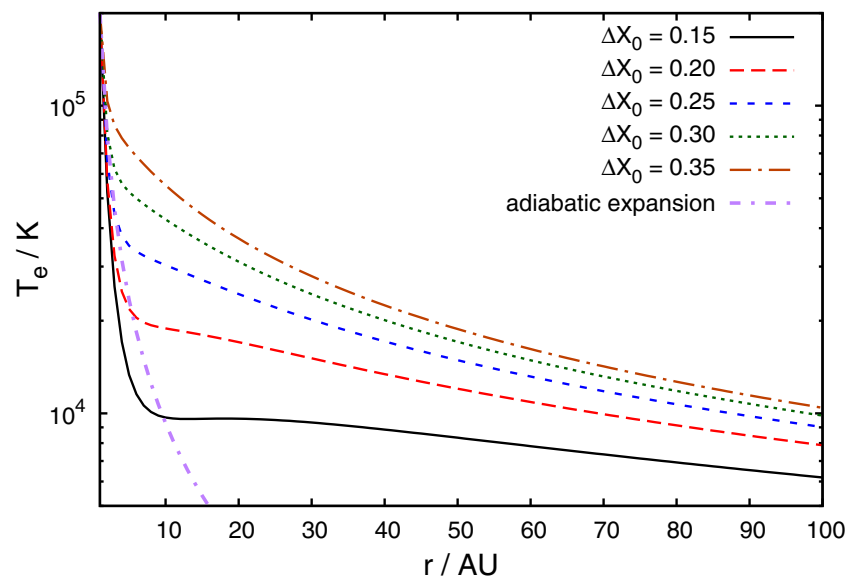

Fig. 4. Electron temperature $T_{\mathrm{e}}$ as a function of distance $r$ from the Sun for five different values of $\Delta X_{0}$ with $r_{0} / L_{\mathrm{j}}=1 / 3$ and $U=400 \mathrm{~km} \mathrm{~s}^{-1}$. The electron temperature beyond $10 \mathrm{AU}$ is higher than predicted from adiabatic expansion.

the flow to the slower regime (i.e., $U_{2}$ ). We estimate the work done per unit time as

$\frac{\mathrm{d} \epsilon(s)}{\mathrm{d} t}=-\frac{\Delta U \cdot \Delta P}{D}=-\Delta U \frac{P_{2}^{*}-P_{1}^{*}}{D}$,

where $D$ is the transit distance over the shock profile, and $P_{1,2}^{*}$ are the adaptive pressures (i.e., the entropized kinetic energy densities) on the upstream and downstream sides of the jump. Especially in the outer heliosphere ( $r \geq 5 \mathrm{AU})$, the ion pressure is dominated by the pick-up-ion pressures on either side of the jump. Under this assumption, the expressions for the ion pressures are significantly simplified (see Fahr et al. 2012) for a perpendicular jump $(\Delta \boldsymbol{U} \perp \boldsymbol{B})$, leading to

$P_{2}^{*}-P_{1}^{*} \simeq P_{1, \text { pui }}\left[\frac{s}{3}\left(2 A_{\perp}(s)+\frac{s^{2}}{A_{\perp}^{2}(s)}\right)-1\right]$,

where the remaining pressure adaptation function $A_{\perp}(s)$ for a perpendicular shock is simply given by $A_{\perp}(s)=s$ with $s \simeq 1+$ $2 \Delta X$. This leads to

$$
\begin{aligned}
P_{2}^{*}-P_{1}^{*} & \simeq P_{1, \text { pui }}\left[\frac{s}{3}(2 s+1)-1\right] \\
& =P_{1, \text { pui }}\left[\frac{1+2 \Delta X}{3}(3+4 \Delta X)-1\right] \approx \frac{10}{3} \Delta X P_{1, \text { pui }},
\end{aligned}
$$

which allows us to formulate the ion-induced energy change as

$$
\begin{aligned}
\frac{\mathrm{d} \epsilon(s)}{\mathrm{d} t} & =-\frac{\Delta U \cdot \Delta P}{D}=-\frac{10}{3} \frac{\Delta U}{D} \Delta X P_{1, \text { pui }} \\
& =-\frac{10}{3} \frac{U \Delta X^{2}}{D} P_{1, \text { pui }} .
\end{aligned}
$$

With this additional term, we now obtain the following corrected differential equation for $\Delta X$ :

$$
\frac{2}{\Delta X} \frac{\mathrm{d} \Delta X}{\mathrm{~d} r}+\frac{1}{n_{\mathrm{e}}} \frac{\mathrm{d} n_{\mathrm{e}}}{\mathrm{d} r}+\frac{2}{r}=-4 \frac{\Delta X^{2}}{L_{\mathrm{j}}}-\frac{20}{3} \frac{P_{1, \text { pui }}}{n_{\mathrm{e}} m_{\mathrm{p}} U^{2} D} .
$$

We write the upstream pick-up-ion pressure in the form $P_{1 \text {,pui }}=$ $\zeta n_{\mathrm{e}} k T_{\text {pui }}$ with the pick-up-ion abundance ratio $\zeta=n_{\text {pui }} / n_{\mathrm{e}}$. This leads to the new differential equation

$$
\frac{2}{\Delta X} \frac{\mathrm{d} \Delta X}{\mathrm{~d} r}+\frac{1}{n_{\mathrm{e}}} \frac{\mathrm{d} n_{\mathrm{e}}}{\mathrm{d} r}+\frac{2}{r}=-4 \frac{\Delta X^{2}}{L_{\mathrm{j}}}-\frac{20}{3} \frac{1}{m_{\mathrm{p}} U^{2} D} \zeta k T_{\text {pui }}
$$


Again, taking $n_{\mathrm{e}} \propto r^{-2}$, this relation then simplifies to

$\frac{2}{\Delta X} \frac{\mathrm{d} \Delta X}{\mathrm{~d} r}=-4 \frac{\Delta X^{2}}{L_{\mathrm{j}}}-\frac{20}{3} \frac{1}{D} \frac{\zeta k T_{\mathrm{pui}}}{m_{\mathrm{p}} U^{2}}$.

We introduce the pick-up-ion Mach number $M_{\text {pui }}^{2} \equiv$ $m_{\mathrm{p}} U^{2} / \zeta k T_{\text {pui }} \simeq 1$ and assume that this number be constant in the outer heliosphere (see, e.g., Fahr \& Ruciński 1999; Fahr 2007). We then find

$$
\begin{aligned}
\Delta X= & \frac{1}{\sqrt{\left(\frac{3 D M_{\mathrm{pui}}^{2}}{5 L_{\mathrm{j}}}+\frac{1}{\Delta X_{0}^{2}}\right) \exp \left[\frac{20}{3} \frac{\left(r-r_{0}\right)}{D M_{\mathrm{pui}}^{2}}\right]-\frac{3 D M_{\mathrm{pui}}^{2}}{5 L_{\mathrm{j}}}}} \\
= & \frac{\Delta X_{0}}{\sqrt{\left(\frac{3 D M_{\mathrm{pui}}^{2}}{5 L_{\mathrm{j}}} \Delta X_{0}^{2}+1\right) \exp \left[\frac{20}{3} \frac{(x-1)}{M_{\mathrm{pui}}^{2}} \frac{r_{0}}{D}\right]-\frac{3 D M_{\mathrm{pui}}^{2}}{5 L_{\mathrm{j}}} \Delta X_{0}^{2}}} .
\end{aligned}
$$

Expansion of the exponential term in Eq. (26) for $D \ll r_{0}$ leads to Eq. (16). Since the shock transit distance is much smaller than $1 \mathrm{AU}$, the corrections due to the pick-up-ion pressure lead to profiles that are qualitatively very similar to the curves shown in Fig. 2.

\subsection{Increased compression due to nonlinear wave steepening}

There may also be processes that counteract those described in Sects. 2.1 and 2.2: processes that support a pile-up of the bulkvelocity jump amplitude. For instance, fluctuations in the bulk velocity may cause such a pile-up by nonlinear superposition. Therefore, we consider wave steepening in the system in addition to the previously discussed processes. Small-scale velocity fluctuations described by $\delta U(x, t)=\delta U(k) \cos [k(x-U t)]$ can pile up into a large-scale fluctuation with $L \simeq L_{\mathrm{j}}=2 \pi / k_{\min }$ by nonlinear wave-coupling and dissipation processes. For onedimensional waves, this situation is described by the following equation (see Infeld \& Rowlands 1990 or Treumann \& Baumjohann 1997):

$\frac{\partial}{\partial t} \delta U+\delta U \frac{\partial}{\partial x} \delta U=F$

where $F$ denotes a dissipation force that counteracts the nonlinear term on the left-hand side and compensates for catastrophic wave steepening and wave breaking. For the so-called Burgers' equation (see Treumann \& Baumjohann 1997), a particular dissipative force is introduced in place of $F$ that is proportional to the second derivative of the velocity perturbation, which leads to the following differential equation:

$\frac{\partial}{\partial t} \delta U+\delta U \frac{\partial}{\partial x} \delta U=\alpha \frac{\partial^{2}}{\partial x^{2}} \delta U$,

where $\alpha$ is a positive dissipation coefficient that acts like a diffusion coefficient (assumed to be constant with distance $r$ ). The background plasma moves with the velocity $U$, and $\delta U$ represents the superposition $\delta U=U+\Delta U$. If the nonlinear steepening of $\delta U$ (second term on the left-hand side of the Burgers' equation, Eq. (28)) increases, the dissipative term on the righthand side can compensate for the nonlinear term and can allow for a stationary solution in the system that co-moves with the nonlinear wave profile. We assume that this developing nonlinear wave asymptotically moves with the velocity $\Delta U$. This allows us to write the Burgers' equation in this particular comoving system, where the first term of the left-hand side disappears (i.e., $\partial \delta U / \partial t=0$ ) when we transform the equation to space coordinates $y=x-\Delta U t$. This procedure then leads to

$(\delta U-\Delta U) \frac{\partial}{\partial y} \delta U=\alpha \frac{\partial^{2}}{\partial y^{2}} \delta U$.

The solution of this equation is easily obtained in the form of a velocity shock ramp given by

$\delta U-\Delta U=-\Delta U \tanh \left(\frac{\Delta U y}{2 \alpha}\right)$,

which can be rewritten in the form

$\delta U=\Delta U\left[1-\tanh \left(\frac{\Delta U y}{2 \alpha}\right)\right]$.

To estimate the appropriate value of $\alpha$ (which has the dimension of $\mathrm{cm}^{2} / \mathrm{s}$ ), we return to the original Burgers' equation and estimate the time scale for steepening (or in the opposite case: for dissolution) of the wave profile by the pure diffusion-type equation (i.e., domination of the dissipation term) given by

$\frac{\partial}{\partial t} \delta U=\alpha \frac{\partial^{2}}{\partial x^{2}} \delta U$

We find the solution of this equation within the system $[-D ;+D]$ by

$\delta U(x, t)=\delta U_{0} \frac{2 D}{\sqrt{4 \pi \alpha t}} \exp \left(-\frac{x^{2}}{4 \alpha t}\right)$.

The kinetic-energy density of the velocity fluctuations within the two flanks $[-D ;+D]$ of such a velocity structure with the structure scale $D$ is given by

$\epsilon_{\mathrm{nl}}=\frac{1}{2 D} \int_{-D}^{+D} \frac{1}{2} m_{\mathrm{p}} n_{\mathrm{e}} \delta U^{2} \mathrm{~d} x$.

Assuming free diffusion, its temporal change is given by

$\dot{\epsilon}_{\mathrm{nl}}=\frac{1}{2 D} \frac{\mathrm{d}}{\mathrm{d} t} \int_{-D}^{D} \frac{1}{2} m_{\mathrm{p}} n_{\mathrm{e}} \delta U^{2} \mathrm{~d} x$.

Taking this expression for $\dot{\epsilon}_{\mathrm{nl}}$ for nonlinear diffusion or steepening per unit volume, we obtain

$$
\begin{aligned}
\dot{\epsilon}_{\mathrm{n} l} & =\frac{1}{2 D} \frac{1}{2} m_{\mathrm{p}} n_{\mathrm{e}} \delta U_{0}^{2} \frac{\mathrm{d}}{\mathrm{d} t}\left[\frac{4 D^{2}}{4 \pi \alpha t} \int_{-D}^{D} \exp \left(-\frac{2 x^{2}}{4 \alpha t}\right) \mathrm{d} x\right] \\
& =D m_{\mathrm{p}} n_{\mathrm{e}} \delta U_{0}^{2} \frac{\mathrm{d}}{\mathrm{d} t}\left[\frac{\sqrt{2 \alpha t}}{4 \pi \alpha t} \int_{-D / \sqrt{2 \alpha t}}^{D / \sqrt{2 \alpha t}} \exp \left(-y^{2}\right) \mathrm{d} y\right] .
\end{aligned}
$$

Evaluating this integral expression further leads to

$$
\begin{aligned}
\dot{\epsilon}_{\mathrm{n} l}= & D m_{\mathrm{p}} n_{\mathrm{e}} \delta U_{0}^{2} \frac{\mathrm{d}}{\mathrm{d} t}\left[\frac{\operatorname{erf}\left(\frac{D}{\sqrt{2 \alpha t}}\right)}{\sqrt{8 \pi \alpha t}}\right] \\
& =D m_{\mathrm{p}} n_{\mathrm{e}} \delta U_{0}^{2}\left[\frac{2 e^{-D^{2} / 2 \alpha t} \frac{\mathrm{d}}{\mathrm{d} t} \frac{D}{\sqrt{2 \alpha t}}}{\pi \sqrt{8 \alpha t}}-\frac{1}{2} \frac{\operatorname{erf}\left(\frac{D}{\sqrt{2 \alpha t}}\right)}{(8 \pi \alpha t)^{3 / 2}} 8 \pi \alpha\right],
\end{aligned}
$$


and finally to

$\dot{\epsilon}_{\mathrm{nl}}=-\frac{D m_{\mathrm{p}} n_{\mathrm{e}} \delta U_{0}^{2}}{\sqrt{8 \pi \alpha t}}\left[\frac{2 \alpha D e^{-D^{2} / 2 \alpha t}}{\sqrt{\pi}(2 \alpha t)^{3 / 2}}+\frac{\operatorname{erf}\left(\frac{D}{\sqrt{2 \alpha t}}\right)}{2 t}\right]$.

According to the profile in Eq. (31), $D \simeq \alpha / \Delta U$. On the other hand, the characteristic time $\tau$ of the shock passage is given by $\tau=D / \Delta U=\alpha / \Delta U^{2}$. Evaluating now the above expression for this characteristic time $\tau$ leads to the following expression:

$\dot{\epsilon}_{\mathrm{nl}}=-\frac{D m_{\mathrm{p}} n_{\mathrm{e}} \delta U_{0}^{2}}{\sqrt{8 \pi D^{2}}}\left[\frac{2 D^{2} \Delta U e^{-D^{2} / 2 D^{2}}}{\sqrt{\pi}\left(2 D^{2}\right)^{3 / 2}}+\Delta U \frac{\operatorname{erf}\left(\frac{D}{\sqrt{2 D^{2}}}\right)}{2 D}\right]$,

or finally (with $\Delta U \approx \delta U_{0}$ as suggested by Eq. (33)) to

$\dot{\epsilon}_{\mathrm{nl}}=-\frac{m_{\mathrm{p}} n_{\mathrm{e}} \Delta U^{3}}{\sqrt{8 \pi} D}\left[\frac{e^{-1 / 2}}{\sqrt{2 \pi}}+\frac{1}{2} \operatorname{erf}\left(\frac{1}{\sqrt{2}}\right)\right]$.

We then obtain the following transport equation with the newly found term for $\dot{\epsilon}_{\mathrm{nl}}$ :

$\frac{1}{r^{2}} \frac{\mathrm{d}}{\mathrm{d} r} r^{2}\left(U \frac{1}{2} n_{\mathrm{e}} m_{\mathrm{p}} \Delta U^{2}\right)=-\frac{3}{2} U k \Delta T_{\mathrm{e}} n_{\mathrm{e}} \frac{\Delta U}{U L_{\mathrm{j}}}-\dot{\epsilon}_{\mathrm{nl}}(\Delta U)$.

The last term on the right-hand side represents the energy that is required to maintain the jump profile. Free diffusion would instead destroy the profile, according to Eq. (35). The transport equation is then given by

$$
\begin{aligned}
\frac{1}{r^{2}} \frac{\mathrm{d}}{\mathrm{d} r} r^{2}\left(U \frac{1}{2} n_{\mathrm{e}} m_{\mathrm{p}} \Delta U^{2}\right) & =-\frac{3}{2} U k \Delta T_{\mathrm{e}} n_{\mathrm{e}} \frac{\Delta U}{U L_{\mathrm{j}}} \\
+ & \frac{m_{\mathrm{p}} n_{\mathrm{e}} \Delta U^{3}}{\sqrt{8 \pi} D}\left[\frac{e^{-1 / 2}}{\sqrt{2 \pi}}+\frac{1}{2} \operatorname{erf}\left(\frac{1}{\sqrt{2}}\right)\right] .
\end{aligned}
$$

With the definition

$\Gamma \equiv\left[\frac{e^{-1 / 2}}{\sqrt{2 \pi}}+\frac{1}{2} \operatorname{erf}\left(\frac{1}{\sqrt{2}}\right)\right] \simeq 0.6$,

we find

$\frac{\mathrm{d}}{\mathrm{d} r}\left(\Delta U^{2} n_{\mathrm{e}}\right)+\frac{2}{r}\left(\Delta U^{2} n_{\mathrm{e}}\right)=-3 \frac{k \Delta T_{\mathrm{e}} n_{\mathrm{e}}}{m_{\mathrm{p}}} \frac{\Delta U}{U L_{\mathrm{j}}}+\frac{2 n_{\mathrm{e}} \Delta U^{3}}{\sqrt{8 \pi} D U} \Gamma$.

Inserting $\Delta T_{\mathrm{e}}$ from Eq. (11) then leads to the following differential equation:

$\frac{\mathrm{d}}{\mathrm{d} r}\left(\Delta U^{2} n_{\mathrm{e}}\right)+\frac{2}{r}\left(\Delta U^{2} n_{\mathrm{e}}\right)=-\frac{4 U^{2} n_{\mathrm{e}} \Delta X^{4}}{L_{\mathrm{j}}}+\frac{2 n_{\mathrm{e}} \Delta U^{3}}{\sqrt{8 \pi} D U} \Gamma$,

or equivalently to

$\frac{\mathrm{d}}{\mathrm{d} r} \Delta X^{2}+\Delta X^{2} \frac{1}{n_{\mathrm{e}}} \frac{\mathrm{d} n_{\mathrm{e}}}{\mathrm{d} r}+\frac{2}{r} \Delta X^{2}=-\frac{4 \Delta X^{4}}{L_{\mathrm{j}}}+\frac{2 \Delta X^{3}}{\sqrt{8 \pi} D} \Gamma$

and

$\frac{2}{\Delta X} \frac{\mathrm{d}}{\mathrm{d} r} \Delta X+\frac{1}{n_{\mathrm{e}}} \frac{\mathrm{d} n_{\mathrm{e}}}{\mathrm{d} r}+\frac{2}{r}=-\frac{4 \Delta X^{2}}{L_{\mathrm{j}}}+\frac{2 \Delta X}{\sqrt{8 \pi} D} \Gamma$.

For a radially symmetric density decrease, we rewrite the transport equation including terms that decrease (first term) and increase (second term) the compression as

$\frac{2}{\Delta X} \frac{\mathrm{d}}{\mathrm{d} r} \Delta X=-\frac{4 \Delta X^{2}}{L_{\mathrm{j}}}+\frac{2 \Delta X}{\sqrt{8 \pi} D} \Gamma$.
Combining decreasing and increasing factors can lead to a vanishing gradient and thus to a constant compression $\Delta X$ if

$\Delta X=\frac{\Gamma}{2 \sqrt{8 \pi}} \frac{L_{\mathrm{j}}}{D} \simeq \frac{0.6}{10} \frac{L_{\mathrm{j}}}{D}=0.06 \frac{L_{\mathrm{j}}}{D}$.

Therefore, the newly derived term for structure steepening will only compete with the first term if the jump amplitude has decreased to values of $\Delta X \lesssim 0.06\left(L_{\mathrm{j}} / D\right)$. Looking for a solution of the full equation, we start from the solution of

$\frac{2}{\Delta X} \frac{\mathrm{d}}{\mathrm{d} r} \Delta X=-\frac{4 \Delta X^{2}}{L_{\mathrm{j}}}$.

As shown in Sect. 2.1, the solution is given by

$\Delta X=\frac{\Delta X_{0}}{\sqrt{1+\frac{4}{L_{\mathrm{j}}} \Delta X_{0}^{2}\left(r-r_{0}\right)}}$

The solution of the other part,

$\frac{2}{\Delta X} \frac{\mathrm{d}}{\mathrm{d} r} \Delta X=\frac{2 \Delta X}{\sqrt{8 \pi} D} \Gamma$,

is derived from

$\frac{1}{\Delta X^{2}} \frac{\mathrm{d}}{\mathrm{d} r} \Delta X=-\frac{\mathrm{d}}{\mathrm{d} r} \Delta X^{-1}=\frac{1}{\sqrt{8 \pi} D} \Gamma$

and yields

$\Delta X^{-1}-\Delta X_{0}^{-1}=-\frac{1}{\sqrt{8 \pi} D} \Gamma\left(r-r_{0}\right)$

This leads to

$\Delta X=\frac{1}{\Delta X_{0}^{-1}-\frac{\Gamma}{\sqrt{8 \pi} D}\left(r-r_{0}\right)}=\frac{\Delta X_{0}}{1-\frac{\Gamma \Delta X_{0}}{\sqrt{8 \pi} D}\left(r-r_{0}\right)}$.

According to these considerations, the general solution is given by the superposition

$\Delta X=\frac{\Omega \Delta X_{0}}{\sqrt{1+\frac{4 r_{0}}{L_{\mathrm{j}}} \Delta X_{0}^{2}(x-1)}}+\frac{\Phi \Delta X_{0}}{1-\frac{\Gamma \Delta X_{0}}{\sqrt{8 \pi}} \frac{r_{0}}{D}(x-1)}$.

The corrections as a result of nonlinear wave steepening are small as long as $\Delta X \gtrsim 0.06$. In those cases, we only need to consider the first term and hence retain the earlier solution we derived in Sect. 2.1 and displayed in Figs. 3 and 4.

\section{Conclusions}

We have shown that traveling solar-wind bulk-velocity jumps effectively process solar-wind electrons in energy at their propagation outward from the Sun through the inner heliosphere. These fluctuations in the solar-wind bulk velocity are ubiquitous, as shown in Fig. 1. In an earlier paper, we have shown that this energization can be expressed in terms of a substantial temperature increase of the solar-wind electrons at larger distances from the Sun of about $50 \mathrm{AU}$ to $90 \mathrm{AU}$. Assuming that the jump amplitude $\Delta X=\Delta U / U$ of the propagating bulk-velocity structure does not change with solar distance $r$, the previous study predicts electron temperatures of more than $10^{5} \mathrm{~K}$ at $90 \mathrm{AU}$ (i.e., at about position of the solar-wind termination shock). In this study, we introduced higher-order corrections that take into account that the energy for the energization of solar-wind electrons 
is taken from the kinetic excess energy of the propagating jump structure. We found that the previous assumption of a constant jump amplitude $\Delta X$ is most probably unrealistic. In addition, the jump structures do permanently work against the ion excess pressure on the downstream side of the shock structure. Taking into account these two physical processes allowed us to quantitatively show that the jump amplitude $\Delta X=\Delta X(r)$ decreases with distance from the Sun, eventually reducing $\Delta X$ independent of the initial value $\Delta X_{0}$ of the jump amplitude to values of $\lesssim 0.1$ at the termination shock, as shown in Fig. 2. The nonlinear pile-up of bulk-velocity fluctuations counteracts these two mechanisms with the tendency to reform the solitary jump structure by forming waves at larger scales. We found, however, that this mechanism is most likely not effective enough to compensate for the reduction of $\Delta X$ with distance, unless $\Delta X \lesssim 0.06$.

Although the described mechanisms reduce $\Delta X$ with distance from the Sun, as shown in Fig. 2, the jump-induced heating mechanism still leads to higher electron temperatures than anticipated from adiabatic cooling at solar distances beyond $10 \mathrm{AU}$. We predict values above $6000 \mathrm{~K}$ to $20000 \mathrm{~K}$ (strongly depending on the initial value of the jump amplitude $\Delta X_{0}$ in the innermost heliosphere at $r=r_{0}=1 \mathrm{AU}$ ) at distances beyond $50 \mathrm{AU}$ with the solar-wind electron-temperature profiles $T_{\mathrm{e}}(r)$ shown in Figs. 3 and 4. Our results agree very well with in situ measurements of the electron-core temperature in the plane of the ecliptic from the Ulysses spacecraft. We achieved the best agreement for values of $\Delta X_{0}$ between 0.25 and 0.3 , which suggests that these values describe the realistic initial jump amplitude in the plane of the ecliptic. In situ observations of the electron temperature are not available for heliocentric distances beyond $5 \mathrm{AU}$, so that our results are a predictive extrapolation beyond the explored range.

We conclude that solar-wind electrons do not rapidly cool with distance from the Sun, as has been generally assumed up to now. They cannot be considered cold beyond $10 \mathrm{AU}$. Instead, they continue to be correlated with solar-wind ion temperatures at large distances (see Richardson et al. 1995).

Acknowledgements. D.V. is supported by NASA grant NNX12AB27G. We used data from NASA's OMNIWeb Service provided by the Goddard Space Flight
Center Space Physics Data Facility (GSFC/SPDF), as well as Ulysses data provided by NASA's National Space Science Data Center (NSSDC).

\section{References}

Achatz, U., Droege, W., Schlickeiser, R., \& Wibberenz, G. 1993, J. Geophys. Res., 98, 13261

Bame, S. J., Phillips, J. L., McComas, D. J., Gosling, J. T., \& Goldstein, B. E. 1992, in Solar Wind Seven Colloquium, Proc. of the 3rd COSPAR Colloquium, Groslar, Germany, eds. E. Marsch, \& R. Schwenn, 139

Breech, B., Matthaeus, W. H., Cranmer, S. R., Kasper, J. C., \& Oughton, S. 2009, J. Geophys. Res., 114, 9103

Chashei, I. V., \& Fahr, H. J. 2014, Sol. Phys., 289, 1359

Echer, E., Gonzalez, W. D., Tsurutani, B. T., et al. 2005, J. Geophys. Res., 110, 2101

Fahr, H. J. 2007, Ann. Geophys., 25, 2649

Fahr, H. J., \& Ruciński, D. 1999, A\&A, 350, 1071

Fahr, H.-J., Chashei, I. V., \& Siewert, M. 2012, A\&A, 537, A95

Feldman, W. C., Asbridge, J. R., Bame, S. J., Montgomery, M. D., \& Gary, S. P. 1975, J. Geophys. Res., 80, 4181

Feng, X. S., Zhang, Y., Sun, W., et al. 2009, J. Geophys. Res., 114, 1101

Gary, S. P., Scime, E. E., Phillips, J. L., \& Feldman, W. C. 1994, J. Geophys. Res., 99, 23391

Infeld, E., \& Rowlands, G. 1990, Nonlinear waves, solitons and chaos (Cambridge: Cambridge University Press)

Janvier, M., Démoulin, P., \& Dasso, S. 2014, A\&A, 565, A99

Lai, H., Jian, L. K., Russell, C. T., et al. 2010, EGU General Assembly Conference Abstracts, 12, 5340

Maksimovic, M., Zouganelis, I., Chaufray, J.-Y., et al. 2005, J. Geophys. Res., 110,9104

McComas, D. J., Bame, S. J., Feldman, W. C., Gosling, J. T., \& Phillips, J. L. 1992, Geophys. Res. Lett., 19, 1291

Pilipp, W. G., Muehlhaeuser, K.-H., Miggenrieder, H., Rosenbauer, H., \& Schwenn, R. 1987, J. Geophys. Res., 92, 1103

Richardson, J. D., Paularena, K. I., Lazarus, A. J., \& Belcher, J. W. 1995, Geophys. Res. Lett., 22, 325

Schlickeiser, R., Jaekel, U., \& Dung, R. 1991, A\&A, 242, L5

Scime, E. E., Bame, S. J., Feldman, W. C., et al. 1994, J. Geophys. Res., 99, 23401

Sokół, J. M., Bzowski, M., Tokumaru, M., Fujiki, K., \& McComas, D. J. 2013, Sol. Phys., 285, 167

Treumann, R. A., \& Baumjohann, W. 1997, Adv. space plasma physics (London: Imperial College Press)

Štverák, Š., Maksimovic, M., Trávníček, P. M., et al. 2009, J. Geophys. Res., 114,5104

Yue, C., \& Zong, Q. 2011, J. Geophys. Res., 116, 12201 